\title{
Tracking heliospheric disturbances by interplanetary scintillation
}

\author{
M. Tokumaru, M. Kojima, K. Fujiki, and M. Yamashita \\ Solar-Terrestrial Environment Laboratory, Nagoya University, Toyokawa, Aichi 442-8507, Japan \\ Received: 18 January 2006 - Revised: 8 May 2006 - Accepted: 8 May 2006 - Published: 1 August 2006
}

\begin{abstract}
Coronal mass ejections are known as a solar cause of significant geospace disturbances, and a fuller elucidation of their physical properties and propagation dynamics is needed for space weather predictions. The scintillation of cosmic radio sources caused by turbulence in the solar wind (interplanetary scintillation; IPS) serves as an effective ground-based method for monitoring disturbances in the heliosphere. We studied global properties of transient solar wind streams driven by CMEs using 327-MHz IPS observations of the Solar-Terrestrial Environment Laboratory (STEL) of Nagoya University. In this study, we reconstructed three-dimensional features of the interplanetary (IP) counterpart of the CME from the IPS data by applying the model fitting technique. As a result, loop-shaped density enhancements were deduced for some CME events, whereas shellshaped high-density regions were observed for the other events. In addition, CME speeds were found to evolve significantly during the propagation between the corona and 1 AU.
\end{abstract}

\section{Introduction}

The Sun emits a continuous supersonic stream of plasma called the solar wind. The solar wind produces the heliosphere, which is a huge bubble in space, extending well beyond the orbit of Pluto. Thus, this supersonic flow engulfs all planets of the solar system, and interacts with them. In the case of the Earth, the solar wind is blocked and deflected by its intrinsic magnetic field, and the magnetosphere is shaped through this interaction.

The large-scale structure of the highly variable solar wind changes drastically over the course of the well-known 11year sunspot cycle. In addition, transient disturbances of

Correspondence to: $\mathrm{M}$. Tokumaru

(tokumaru@ stelab.nagoya-u.ac.jp) the solar wind are sometimes generated by eruptive phenomena in the solar atmosphere. These solar wind variations affect the space environment around the planets, and they sometimes cause significant disturbances in the magnetosphere or upper atmosphere of the Earth. Such space disturbances cause adverse conditions for space-borne systems and astronauts. In the worst case, spacecraft are lost due to unrecoverable failures, and human health is endangered. Thus, solar-driven disturbances are a serious threat for human advances into space. Ground-based technological systems such as radio communications, power facilities, navigations, which are fundamental services for our life, are also vulnerable to space disturbances. In order to minimize the loss of space-borne or ground-based systems, a precise prediction of the time-variable conditions in the space environment (so-called "space weather prediction") is needed in the near future (Marubashi, 1989; Luhmann, 1997).

Among various kinds of solar activities, coronal mass ejections (CMEs) are known to cause significant space disturbances (Gosling, 1993; Lindsay et al., 1995). In this sense, CMEs are the primary targets for space weather predictions (Luhmann, 1997). A CME is a sudden release of dense plasma and strong magnetic flux into interplanetary space. It is usually identified from coronagraphic observations as a transient bright feature moving outward from the sun. Typical values of mass and energy for a single CME event are $10^{15}-10^{16} \mathrm{~g}$ and $10^{30}-10^{31} \mathrm{erg}$, respectively (St. Cyr et al., 2000; Yashiro et al., 2004). CME speeds range from several $10 \mathrm{~s} \mathrm{~km} / \mathrm{s}$ to $>2000 \mathrm{~km} / \mathrm{s}$, and the mean value is $\sim 450 \mathrm{~km} / \mathrm{s}$ (St. Cyr et al., 2000; Yashiro et al., 2004). It should be noted here that these are projected speeds. Fast CMEs drive shock waves in the solar wind, and CME-driven shocks accelerate energetic particles (Reames, 1999), which are hazardous for spacecraft instruments and astronauts. When CMEs impact the Earth's magnetosphere, they can give rise to geomagnetic disturbances (Lindsay et al., 1995; St. Cyr et al., 2000), which can have detrimental effects on space-borne

Published by Copernicus GmbH on behalf of the European Geosciences Union and the American Geophysical Union. 
(a)

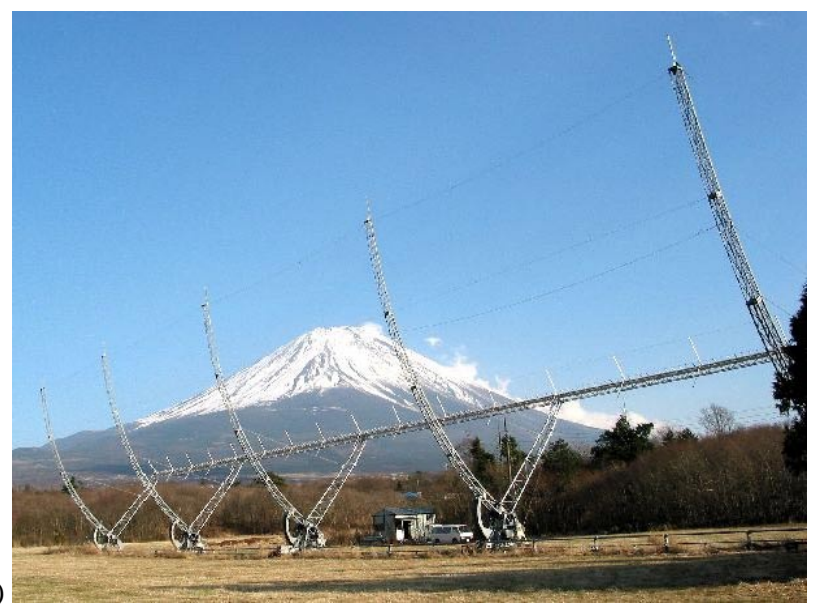

(b)

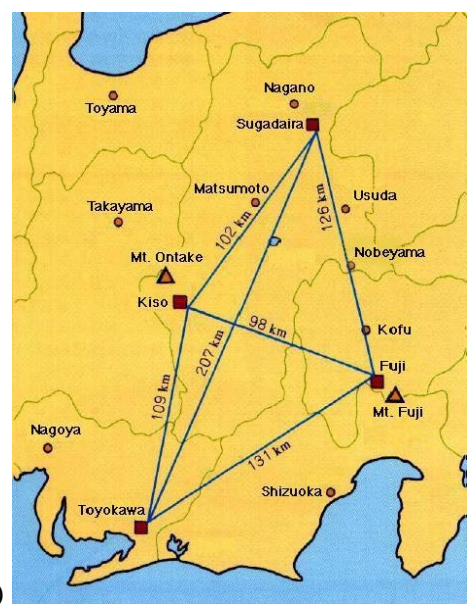

Fig. 1. (a) The 327-MHz radiotelescope at Fuji Observatory, and (b) geographic locations of STEL four-station system.

and ground-based systems via energetic particles and fluctuating magnetic fields.

The 3-dimensional structure and propagation dynamics of CME are the fundamental knowledge required to predict space weather from observational data near the Sun. However, they are poorly understood owing to the lack of observational data. In particular, CME observations in the solar wind are rather sparse, and the global properties of CME between the Sun and the Earth orbit are still largely obscure. We have been addressing this problem with observations of interplanetary scintillation (IPS; Tokumaru et al., 2000, 2003a, b, 2005a, b, c). IPS serves as an effective ground-based method to observe the solar wind (e.g. Hewish et al., 1964; Hewish, 1989). IPS observations are particularly useful to study the global properties of transient streams associated with CMEs, since they allow us to probe a large volume of the inner heliosphere in a short time (Gapper et al., 1982; Watanabe and Schwenn, 1989).

This paper reports recent results from our IPS study of CMEs. The outline of this paper is as follows. In Sect. 2, we describe solar wind observations made with the $327-\mathrm{MHz}$ IPS system of the Solar-Terrestrial Environment Laboratory (STEL) of Nagoya University. In Sect. 3, we describe an analysis used to retrieve the 3-dimensional distribution of a CME from our IPS data. In Sect. 4, we present some results obtained from our IPS observations. In Sect. 5, we summarize and discuss the results.

\section{Interplanetary scintillation measurements of the solar wind}

Since the solar wind is a turbulent medium, small-scale density irregularities are ubiquitous in the heliosphere. Radio waves from cosmic sources are scattered by these density irregularities, and rapid fluctuations of the signal intensity are observed on the ground. This phenomenon is called inter- planetary scintillation, in short IPS (Hewish et al., 1964). IPS has been employed as a useful remote-sensing probe for the solar wind plasma since its discovery. We can derive a bulk flow speed of the solar wind by detecting a time difference between the IPS diffraction patterns observed at two separated stations (Dennison and Hewish, 1967). We can also estimate solar wind density fluctuation levels along a line-ofsight from observed IPS strength, if wave scattering is weak (Hewish and Symonds, 1969). In addition, power spectra of IPS provide information on the solar wind density turbulence (Readhead et al., 1978).

If we observe IPS for many radio sources in a day, we can produce a sky projection map of the solar wind on a daily basis. Such mapping observations by IPS are quite suitable for detecting and tracking solar wind disturbances between the Sun and the Earth orbit. This unique IPS capability was first demonstrated by observations with the Cambridge $36000 \mathrm{~m}^{2}$ array at a frequency of $81.5 \mathrm{MHz}$ (Gapper et al., 1982). Although IPS observations there have been discontinued, the utility of IPS mapping observations for studying IP disturbances has been demonstrated by research groups in Russia (Vlasov, 1979), India (Manoharan et al., 2001) and Japan (Tokumaru et al., 2000). These IPS facilities use higher frequencies, enabling observations closer to the Sun.

Our IPS observations have been carried out regularly since the early 1980s using the 327-MHz multi-station system (Kojima and Kakinuma, 1990; Asai et al., 1995). Figure 1 shows the geographic location of the STEL IPS system and one of the IPS antennas (the $327-\mathrm{MHz}$ radio-telescope at Fuji Observatory). Each IPS antenna has a large collecting area $\left(\sim 2000 \mathrm{~m}^{2}\right)$ to detect faint signals from weak sources. The available scintillation sources changes as the Sun moves against the background from day to day and those with solar elongation less than $90^{\circ}$ are selected for observation. In our IPS observations, 30-40 sources are observed daily between April and December of every year. IPS observations 
2000/07/11:22 UT- 07/12: 7 UT
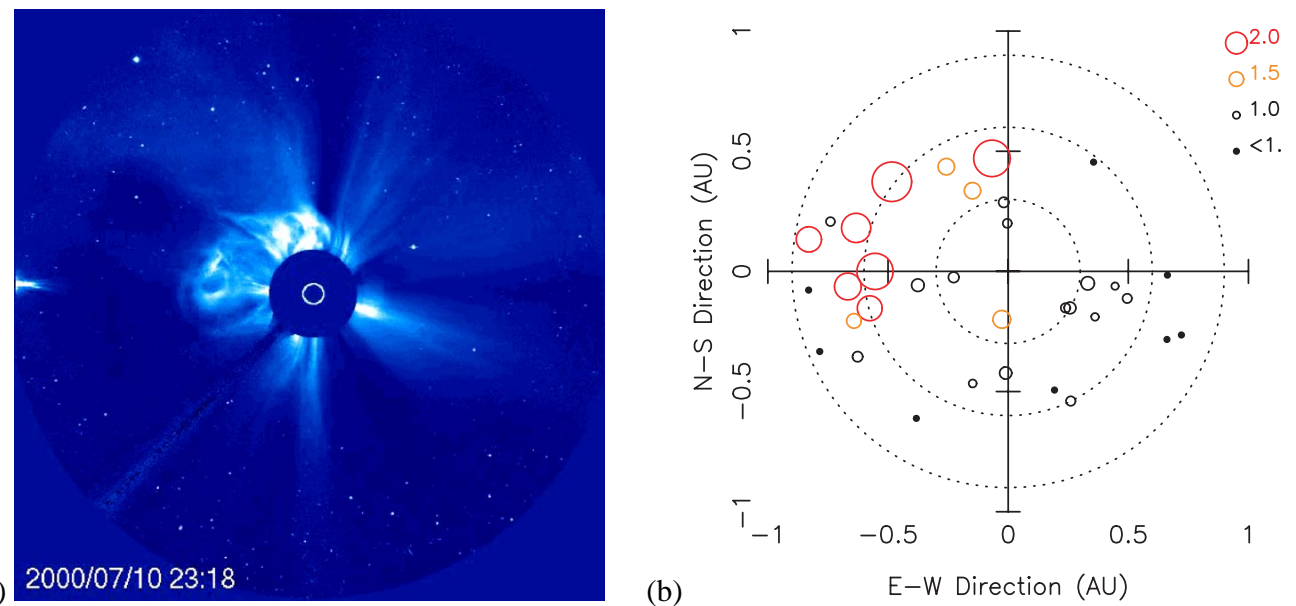

Fig. 2. (a) SOHO/LASCO image for the CME event on 10 July 2000 (from http://lasco-www.nrl.navy.mil/), and (b) sky-projection map of g-value obtained from our IPS observations between 11 July 2000, 22:00 UT and 12 July 2000, 07:00 UT. The center of the g-value map corresponds to the location of the Sun, and dotted concentric circles are constant $R_{S E} \sin \varepsilon(\mathrm{AU})$ contours drawn every $0.3 \mathrm{AU}$, where $\varepsilon$ is the solar elongation angle and $R_{S E} \sim 1 \mathrm{AU}$. Circles indicate line-of-sight locations where g-values were measured, and size and color represent the strength of g-value.

are not conducted during the months January to March owing to heavy annual snowfall in the mountainous regions where the IPS antennas are located. Solar wind speeds and scintillation levels are derived from our IPS observations.

We show an example of our IPS observations for a CME event in Fig. 2. A white-light image of a CME observed by the LASCO C3 coronagraph (Bruecker et al., 1995) is shown in the left panel of Fig. 2. As shown here, a bright CME was observed on the northeast limb on 10 July 2000 . The right panel of Fig. 2 is a sky projection map produced from our IPS observations between 11 and 12 July 2000 . The center of this map corresponds to the location of the Sun, and the dotted circles are constant $R\left(\equiv R_{S E} \sin \varepsilon\right)$ contours drawn every $0.3 \mathrm{AU}$ (where $R_{S E}$ and $\varepsilon$ are the Sun-Earth distance and the solar elongation angle, respectively). In this map, density disturbance factors, so-called g-values (Gapper et al., 1982), derived from our IPS observations are indicated by circles. Color and size of the circles represent the strength of $\mathrm{g}$-values. The g-value is a measure of the scintillation level for a line-of-sight of a given source, and is related to solar wind density fluctuations $\Delta N_{e}$ integrated along the line-ofsight when wave scattering is weak. Note that the small-scale turbulence level of $\Delta N_{e}$ is roughly proportional to the solar wind electron density $N_{e}$ (Armstrong and Coles, 1978): $N_{e} \propto \Delta N_{e}$. In the case of $327-\mathrm{MHz}$, the weak scattering condition holds for $R>0.2 \mathrm{AU}$, where $R$ is the radial distance from the Sun. The g-value is normalized to the turbulence level of the quiet solar wind, so that $g>1$ means an excess of $\Delta N_{e}$. When enhanced density fluctuations associated with CMEs intersect a line-of-sight, we obtain an abrupt increase in g-value. As shown in Fig. 2, marked enhancements in g-value were observed at radial distances between 0.4 and $0.6 \mathrm{AU}$ in the northeast quadrant about one day after the occurrence of the CME event in the corona. These g-value enhancements are considered as an interplanetary counterpart of the 10 July 2000 CME event. It should be noted that the distribution of enhanced g-values in the map is in good agreement with the radial extension of brightness enhancements in the LASCO image. Accompanying the g-value enhancements were distinct increases in the solar wind speeds derived from our IPS observations.

We show another example of CME observations made with the STEL IPS system in Fig. 3. Sky projection g-value maps produced from our IPS observations for 4 consecutive days (between 5 and 9 June 2000) are indicated in the middle panel of Fig. 3. The upper two panels show solar soft $\mathrm{X}$-ray intensity and energetic proton fluxes measured by the GOES geosynchronous satellite, and the lower two panels show the solar wind density and speed measured by the ACE spacecraft at the L1 point (McComas et al., 1998). A fast CME event occurred on 6 June 2000, in association with an $\mathrm{X} 2.3 / 3 \mathrm{~B}$ flare and a solar proton event. This CME event exhibited a circular shape of a bright material surrounding the Sun and propagating outward in all directions. Such an event, which is called a halo CME, is interpreted as a CME directed to the Earth. An IP shock driven by this halo CME was observed by ACE on 8 June 2000 at 09:00 UT. As shown in Fig. 3, clear increases in the g-values, ascribed to an IP disturbance associated with the halo CME, were observed on 7-9 June several hours before the arrival of the IP shock at ACE. Solar wind velocity increases (from $\sim 400 \mathrm{~km} / \mathrm{s}$ to $\sim 600 \mathrm{~km} / \mathrm{s}$ ) were also detected from our IPS observations 
2000/06/05: 15 UT -2000/06/09: 15 UT

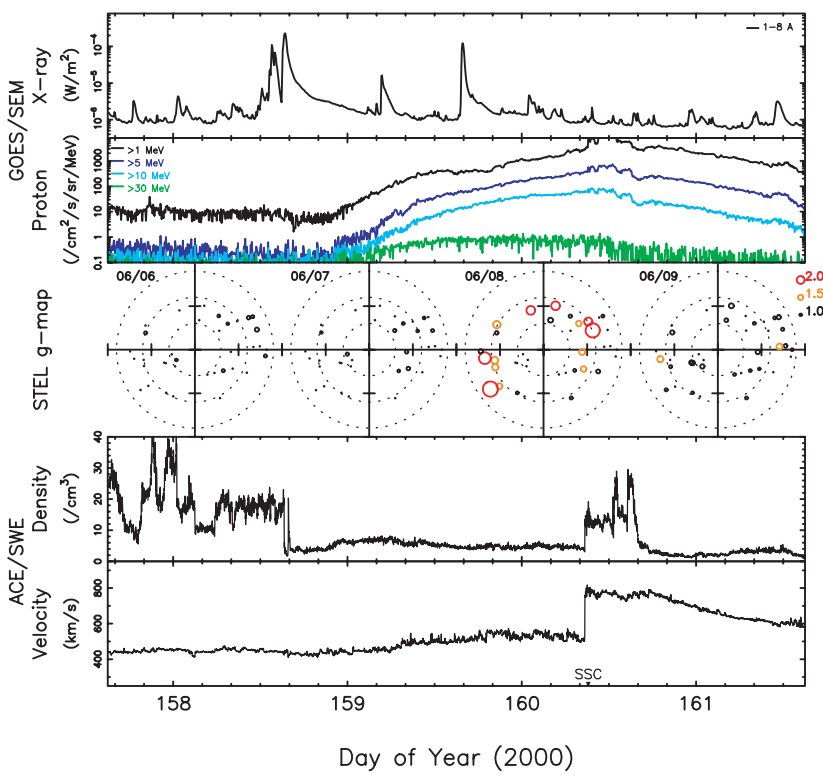

Fig. 3. (From top to bottom) Solar soft X-ray flux and energetic $(>1,>5,>10$, and $>30 \mathrm{MeV}$ ) proton flux observed by the GOES-8 satellite (from http://spidr.ngdc.noaa.gov), sky-projection g-value maps produced from STEL IPS observations, solar wind density and speed measured by ACE spacecraft (from http://www. srl.caltech.edu/ACE/ASC/level2/index.html) for the period between 5 June 2000, 15:00 UT and 9 June 2000, 15:00 UT. The onset of an SSC (sudden storm commencement) is indicated in the bottom panel.

during the same period. This velocity jump is smaller than that associated with the IP shock, however it is not inconsistent with the ACE observations since IPS speeds include the effect of line-of-sight (LOS) integration. An important point to note on our IPS data is that enhanced g-values form a ring-shaped distribution encircling the Sun in the map. This feature is consistent with the fact that the event was Earthdirected.

The IPS sky projection maps thus reveal information on the 3-dimensional properties (e.g. radial distance, angular extent, propagation direction) of the CME in the solar wind. However, attention must be paid to the inherent line-of-sight (LOS) integration in IPS observations, which can significantly affect the apparent features. Therefore, we need to remove the integration effect carefully in order to elucidate the CME's 3-dimensional properties in more detail.

\section{Model fitting analysis of IPS observations}

We fit a 3-dimensional model of the solar wind, which includes a transient component, to our IPS observations in order to remove the LOS integration effect. We briefly describe the method here (a detailed description is presented in Toku- maru et al., 2003a). In this analysis, we exclusively used g-value data derived from our IPS observations, since an analytical method to deconvolve the solar wind velocity data of transient events is not yet available.

For weak scattering, the g-value is given by the following equation

$g^{2}=\frac{1}{K} \int_{0}^{\infty} \Delta N_{e}^{2} w(z) d z$,

where $z$ is a distance along the line-of-sight, and $w(z)$ is the IPS weighting function (Young, 1971) which is given by

$w(z)=\int_{0}^{\infty} k^{1-q} \sin ^{2}\left(\frac{k^{2} z \lambda R F}{4 \pi}\right) \exp \left(-\frac{k^{2} z^{2} \Psi^{2}}{2}\right) d k$

Here, $k, q, \lambda_{R F}$, and $\Psi$ are the spatial wavenumber and the spectral index of density turbulence, the wavelength for the observing frequency $(0.92 \mathrm{~m}$ for $327 \mathrm{MHz})$, and the apparent angular size of the radio source, respectively. The intrinsic value of $\Psi$ is different for each source, however accurate information on it is unavailable for all sources used in our IPS observations. Therefore, we assumed here $\Psi=0.1$ arcsecond, which is a typical value of a scintillation source at 327 MHz. Note that more realistic values of $\Psi$ would not produce any significant changes in the analysis, since model calculations of the g-value are insensitive to $\Psi$. The solar wind turbulence is known to be well described by the Kolmogorov spectrum $(q=11 / 3)$ for all distances except for the vicinity of the Sun (Woo and Armstrong, 1979). Therefore, we assumed $q=11 / 3$ in the analysis. We also assumed $\Delta N_{e}=f_{N_{e}} \Delta N_{e 0}$, where $\Delta N_{e 0}, f_{N_{e}}$ are the density fluctuation level of the ambient solar wind and a dimensionless parameter to represent the deviation (either excess or deficit) from the ambient level, respectively. In Eq. (1), $K$ is a normalization factor, which is given by $K \equiv \int_{0}^{\infty} \Delta N_{e 0}^{2} w(z) d z$.

Provided that a suitable model for the 3-dimensional distribution of $\Delta N_{e}$ in the solar wind is given, the g-value can be calculated by using Eqs. (1) and (2). This fact enables us to determine a realistic $\Delta N_{e}$ model by comparing between calculated and observed g-values. The basic idea to provide observational constraints on a model of IP disturbances by using g-value data was first proposed by Tappin (1987). In his study, a shell-shaped $\Delta N_{e}$ enhancement model which assumed an isotropic angular extent was used. The $\Delta N_{e}$ model fit to our g-value data is similar to the one used by Tappin (1987), but with some distinct differences. For example, our $\Delta N_{e}$ model allows for anisotropy of the angular extent and angular dependence of the propagation speed, thereby more closely simulating the global features of an enhanced $\Delta N_{e}$ region.

To fit IPS data for CME events, we employed a $\Delta N_{e}$ model, which included a localized enhancement $\left(f_{N_{e}}\right)$ above an isotropic radial gradient of the background level $\left(\Delta N_{e 0}\right)$. In our analysis, the $\Delta N_{e}$ enhanced region was assumed to have a Gaussian form variation, which was defined by an 
e-folding radial thickness $D$, an e-folding major angular extent $\theta_{0}$, a ratio of minor-to-major angular extent $A R$, an inclination of the maximum angular elongation $\beta$ (defined in the plane of sky in a counterclockwise sense), a peak value $C_{1}$, and the heliographic coordinates of the central axis $\left(\lambda_{0}\right.$, $\phi_{0}$ ). For small $D$ and $A R \sim 1$, our model represents a shell or bubble-shaped CME structure, and $A R<1$ means an elongated structure in a direction defined by $\beta$. The $\Delta N_{e}$ enhanced region was also assumed to expand radially at a given speed $V_{S}$ during IPS observations. It has been reported from earlier studies that the maximum expansion speed of an IP shock or disturbance is likely to occur in the radial direction above the flare site (Smart and Shea, 1985). In this analysis, we use the following function to account for the angular dependence of the expansion speed

$V_{S}=V_{S 0} \cos ^{\alpha}(\theta / 2)$,

where $\alpha$ and $\theta$ are the index of curvature and the separation angle with respect to the center axis of the $\Delta N_{e}$ enhanced region, respectively. As for the background component, $\Delta N_{e 0}$ is assumed to be distributed as $R^{-2}$, and this is validated by many observational studies (e.g. Hewish and Symonds, 1969; Armstrong and Coles, 1978).

We calculated the g-values with Eqs. (1) and (2) and the $\Delta N_{e}$ model by considering the geometry of IPS observations for a given day, and optimized the model to measured g-values by adjusting nine parameters: $D, \theta_{0}, A R, \beta, C_{1}, \lambda_{0}$, $\phi_{0}, V_{S 0}$, and $\alpha$. The optimization was done by minimizing the rms deviation $\sigma$ given by

$$
\sigma^{2}=\frac{1}{N} \sum_{i=1}^{N} \frac{\left(g_{\text {obs }}-g_{\text {cal }}\right)^{2}}{g_{\text {obs }}^{2}}
$$

where $g_{\text {obs }}$ and $g_{\text {cal }}$ are the observed and calculated g-values, respectively, and $N$ is the number of observations. We used the Simplex method to search for the minimum. The Simplex method is an effective minimizing algorithm for general nonlinear functions without using derivatives, and it attempts to enclose the minimum inside an irregular volume defined by a "simplex" (i.e. a polyhedron in n-dimensional space, one dimension for each parameter defining the model). The simplex size is continuously changed and mostly diminished so that finally it is small enough to contain the minimum with the desired accuracy. The convergence criterion used in the analysis is that the change in $\sigma^{2}$ at each iteration is within a fractional tolerance level of $10^{-6}$. A few hundred iterations are typically needed for convergence.

The sharpness of the $\sigma^{2}$ variation around the minimum can be used as a measure of the estimation error for a given parameter. The estimation errors in the analysis depend on the observation geometry (the heliocentric distance and the propagation direction of CMEs) as well as goodness of the fit. In the case of IPS observations of an Earth-directed CME at distances $0.5-0.8 \mathrm{AU}$ (the most frequent case in this analysis, see the next section), the coordinate of the central axis $\left(\lambda_{0}, \phi_{0}\right)$ and the angular extent anisotropy $A R$ are best determined among the model parameters. Typical values of the estimation errors for the coordinates and $A R$ are $\sim 5^{\circ}$ and $\sim 0.01$, respectively, as far as a high degree of the correlation is obtained. In comparison, estimation of the inclination angle $\beta$ is somewhat crude, with typical errors being $5-10^{\circ}$. The estimation errors of these parameters, particularly that of longitude, increase with increasing separation angle between the central axis and the Sun-Earth line. Note that $A R$ and $\beta$ are hardly determined for the case of broadside observation geometry. The estimation errors of the propagation speed $V_{S O}$ are usually small, typically a few $\%$ even for the halo geometry. On the other hand, the halo geometry is not suitable to obtain reliable estimates of the angular extent $\theta_{0}$ and the enhancement factor $C_{1}$, since these parameters are coupled. Typical values of the estimation error for $\theta_{0}$ and $C_{1}$ are $10-20^{\circ}$ and $\sim 30 \%$, respectively, for the halo geometry. The estimation errors of the radial thickness $D$ are typically $0.01-$ $0.03 \mathrm{AU}$. The estimates of the curvature index $\alpha$ are usually associated with large errors, $\sim 0.5$, although this parameter is essential to obtain a good fit for some events.

As mentioned above, the model used here is a rather simple one which assumes a smooth variation with a single peak. It is obvious that the current version of the model fitting analysis can not deal with IPS data which include either irregular structures or multiple components of IP disturbances. Nevertheless, we think that this analysis is useful to determine large-scale properties of an isolated CME event.

\section{Results}

Remote-observer views of the best fit model for some CME events are displayed in Fig. 4. Detailed descriptions of the analysis for these CME events are presented in Tokumaru et al. (2003a, b, 2005b, c). An iso- $\Delta N_{e}$ counter of the enhancement component for a given level is plotted here. It should be noted that the $R^{-2}$ gradient of the background component is removed in this figure. The origin of each plot corresponds to the location of the Sun, and the x-axis is directed to the Earth. The length of each axis represents 1 AU. These models are thought as a first-order approximation of global features of CMEs in the solar wind, and they are found to be generally consistent with white-light or in situ measurements of CMEs, as discussed in other studies (Tokumaru et al., 2003a, b, 2005b, c). An important point to note is that there is a variety of the angular extent; some of CME events had an elongated structure $(A R<1)$, and some had a shell-shaped structure with a nearly isotropic angular extent $(A R \sim 1)$. This aspect of interplanetary CMEs was not fully appreciated from earlier observational studies. An accurate description of the global morphology is clearly essential for improving space weather predictions.

We have analyzed our IPS data for 16 CME events including those shown in Fig. 4. The analyzed CME events 


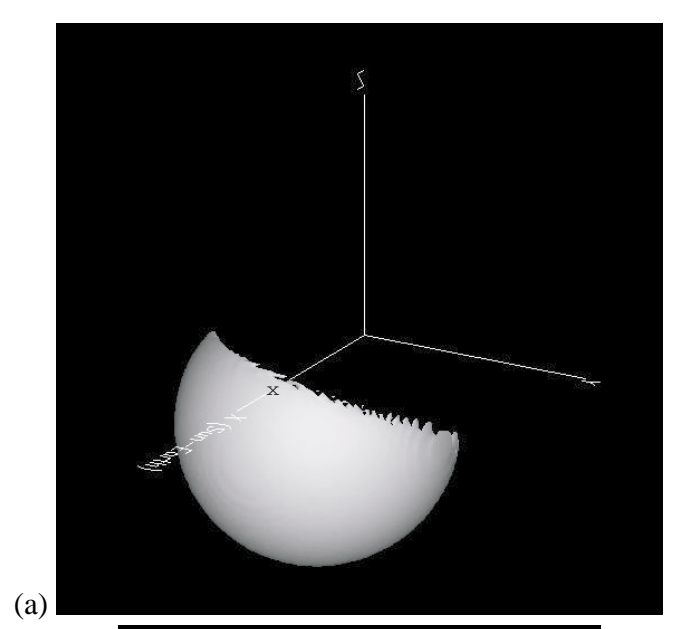

(a)

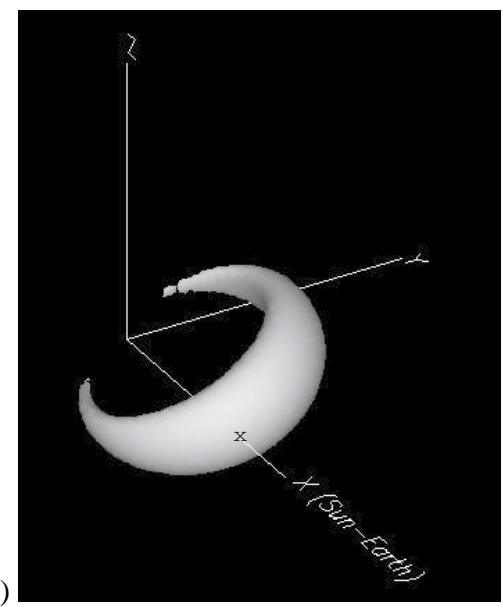

(b)

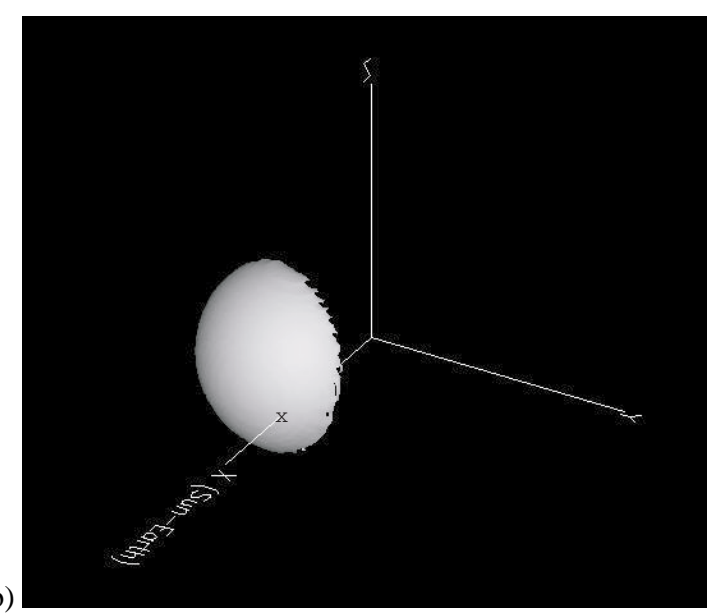

(d)

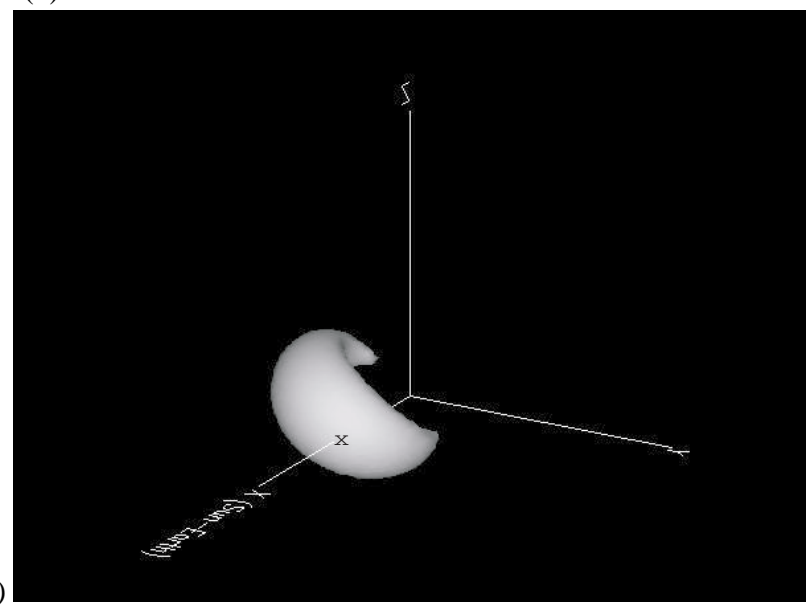

Fig. 4. Remote-observer's views of the best fit model determined for (a) 20 September 1999, (b) 10 July 2000 , (c) 14 July 2000 (adopted from Tokumaru et al., 2003a), and (d) 28 October 2003 CME events. The solar ecliptic coordinate system is used here, and the xy-plane is the ecliptic. The heliographic coordinates of the central axis for (a-d) are S35E04, N13E21, N11W17, and N15E15, respectively. The intersection point of the outer shell/loop of the IP disturbance with the x-axis is marked by a cross in each panel.

occurred between 1998 and 2003, and they were selected by the following two criteria; (1) g-value enhancements with $g>1.5$ were observed for several lines-of-sight in association with the occurrence of a CME observed with LASCO. Since no LASCO observations were available during JulySeptember in 1998, we identified the CME occurrence from in situ observations of the magnetic flux rope at $1 \mathrm{AU}$ during this period. Most of the events selected here were associated with halo or partial halo CMEs. This is attributed to the fact that our IPS observations have greater sensitivity for Earth-directed CMEs than those over the limb. Strictly speaking, CMEs propagating in an oblique direction with respect to the Sun-Earth line are best observed by IPS, and the maximum sensitivity for a given direction depends on the radial distance. (2) A significant correlation between observed and calculated g-values was obtained by the analysis: i.e. correlation coefficients $\rho>0.5$. Since the model fitted to observations is rather simple, many CME events with a more complex structure were excluded by this criterion.
The best fit parameters $V_{S 0}, \alpha, \lambda_{0}, \phi_{0}, D, \theta_{0}, A R, \beta, C_{1}$ determined for these CME events are listed in Table 1. The parameters $R$ and $\rho$ in the table correspond to heliocentric distances of $\Delta N_{e}$ peak and correlation coefficients between observed and calculated g-values, respectively. The latter is a measure of goodness of the fit, and used to select the CME events, as mentioned above. Heliographic locations of associated flare, separation angles between the flare site and the central axis $\xi$, plane-of-sky speeds of CME measured by LASCO $V_{\mathrm{CME}}$, and the average Sun-Earth transit speeds $V_{1} \mathrm{AU}$ are also shown in the table. The $V_{\mathrm{CME}}$ data were taken from the SOHO/LASCO CME catalog (Yashiro et al., 2004), and the $V_{1} \mathrm{AU}$ data were determined from the time difference between the CME occurrence and the IP shock arrival at $1 \mathrm{AU}$. Mean values of $V_{\mathrm{CME}}$ and $V_{1 \mathrm{AU}}$ for all events were $1264 \mathrm{~km} / \mathrm{s}$ and $1036 \mathrm{~km} / \mathrm{s}$, respectively. Thus, high-speed events were selected in this study. This selection bias may come from the fact that fast CME events can effectively give rise to $\Delta N_{e}$ enhancements in the solar wind. 
Typical features of interplanetary CMEs deduced from the analysis results are summarized below. Here, one must be reminded of a bias due to the event selection effect.

- An elongated $(A R<0.35)$ structure was determined for about $60 \%$ of analyzed events. The major angular span of the elongated structure was about 4 times (on an average) greater than that of the minor one. The structure tended to elongate in the longitudinal direction, while a large inclination $\left(|\beta|>45^{\circ}\right)$ was observed for some CME events that occurred in 1999. For the isotropic events, a mean value of the angular span was about $50^{\circ}$.

- The radial thickness was small, $D \sim 0.1 \mathrm{AU}$, and there was no significant difference in $D$ between elongated and isotropic events. This thickness is consistent with a typical size of the IP shock thickness at $1 \mathrm{AU}$ (Borrini et al., 1982).

- The mean value of the peak enhancement factor was about 7 for all analyzed events, and no significant difference was found between elongated and isotropic events. Provided that $N_{e} \propto \Delta N_{e}$ and the ambient solar wind density is $\sim 5 / \mathrm{cc}$ at $1 \mathrm{AU}$, this peak enhancement factor corresponds to $\sim 35 / \mathrm{cc}$.

- The central axis of the best fit model $\left(\lambda_{0}, \phi_{0}\right)$ differed significantly from that of the associated flare site for some events, and an mean angular separation between the flare site and the inferred central axis is $36^{\circ}$, larger than the estimation errors. This means that the center of an interplanetary CME does not necessarily lie above the flare site. No systematic shift was found in either longitude or latitude.

- The propagation speeds $V_{S 0}$ showed large diversity, ranging between $\sim 500 \mathrm{~km} / \mathrm{s}$ and $>1500 \mathrm{~km} / \mathrm{s}$ (average $\sim 970 \mathrm{~km} / \mathrm{s}$ ). When these speed data are compared with $V_{C M E}$ and $V_{1 A U}$, some CMEs are found to decelerate significantly during propagation. Other CMEs show insignificant change of propagation speed or some acceleration between the Sun and the Earth orbit.

- A mean value of $\alpha$ was about 2, although derived values ranged between 0 and 5.5, and this is in excellent agreement with the empirical model $(\alpha=2)$ derived from a statistical study of IP shocks (Smart and Shea, 1985). There was no significant difference between elongated and isotropic events.

\section{Summary and discussion}

STEL IPS observations were analyzed to study the global features of interplanetary CMEs, which is one of the pieces of key information needed to improve space weather predictions. We reconstructed the 3-dimensional distribution of
CME-generated $\Delta N_{e}$ enhancements in the solar wind from our g-value data by using the model fitting method. As a result, we successfully fit a 3-dimensional model of $\Delta N_{e}$ distribution to our IPS observations for 16 selected CME events for the period 1998 to 2003. Some of the best fit models suggested that the CMEs were elongated in a given direction, being loop-shaped. Other models suggested that the CMEs had shell-shaped distribution with a nearly isotropic angular extent. The maximum angular extent of the elongated structure tended to occur in a longitudinal direction, while large inclinations $\left(>45^{\circ}\right)$ were observed for the CME events in 1999. The radial thickness of the model density enhancement was usually small, and generally consistent with a typical scale of the density compression region associated with IP shocks at $1 \mathrm{AU}$. Evolution of CME propagation speeds in the solar wind was also disclosed by comparing our IPS observations with coronal and/or near-Earth observations. Some of the data suggested that the CMEs were significantly decelerated during propagation between the Sun and the Earth orbit. Other data indicated that the CMEs propagated at an almost constant speed, or gradually accelerated during propagation. The mean value of $\alpha$, which controls the angular dependence of the propagation speed, was found to be nearly equal to the one used in the empirical model of the IP shock, although measured $\alpha$ showed a large scatter.

Following earlier studies (e.g. Watanabe and Schwenn, 1989; Moore and Harrison, 1994; Manoharan et al., 2001), we used $\Delta N_{e}$ enhancements observed by IPS as a proxy for interplanetary CMEs. However, we should keep in mind that the origin of $\Delta N_{e}$ enhancements is still an unsettled question. That is, there are two possible interpretations for $\Delta N_{e}$ enhancements; one is the shocked solar wind plasma (i.e. the energized ambient solar wind) and the other is a core plasmoid of coronal origin (so called "coronal ejecta"). IPS observations cannot discriminate between these two unless one carefully examines their correspondence with other complementary observations for a CME event.

It was reported from a comparison study using Cambridge IPS observations and in situ data that a majority of g-value enhancements were associated with the compression region between the transient high-speed flow and the IP shock driven by a CME (Moore and Harrison, 1994). The global features revealed from our IPS observations are found to be generally consistent with characteristics of the shocked plasma for most of the cases. In particular, the shellshaped $\Delta N_{e}$-enhanced regions are well explained by a highdensity plasma generated at the shock front through interaction between a fast CME and a nearly uniform ambient solar wind. The loop-like density-enhanced structure also can be explained by the shocked plasma provided that the ambient solar wind structure is bimodal. The solar wind is composed of fast and slow speed components, and the shocked plasma is expected to develop predominantly in the slow wind, since strong compression takes place there. Therefore, an elongated structure of $\Delta N_{e}$ enhancements is likely 
Table 1. Model parameters for $16 \mathrm{CME}$ events. $V_{S 0}, \alpha$, coordinate of the center axis (longitude and latitude), $D, \theta_{0}, A R, \beta, C_{1}$ and correlation coefficients $\rho$ determined from the g-value data are shown. Heliographic locations of the associated flare, angular separations between the flare site and the central axis $\xi$, heliocentric distances $R$ are also shown in this table. The dates of CME occurrence are used except for the cases of 24 August and 23 September 1998 events when coronagraph observations by the SOHO spacecraft were unavailable. Instead, flare occurrence dates are used for these cases. Plane-of-sky speeds of CME $V_{\mathrm{CME}}$ (from http://cdaw.gsfc.nasa.gov/CME_list/ index.html), heliographic coordinates of associated flare site, and Sun-Earth transit speeds of IP shock $V_{1}$ AU are included in this table for comparison. See [1] Tokumaru et al. (2000), [2] Tokumaru et al. (2003a), [3] Tokumaru et al. (2003b), [4] Tokumaru et al. (2005b), [5] Tokumaru et al. (2005c).

\begin{tabular}{|c|c|c|c|c|c|c|c|c|c|c|c|c|c|c|c|}
\hline Date & $\begin{array}{l}\text { Flare } \\
\text { Site }\end{array}$ & $\begin{array}{c}V_{\mathrm{CME}} \\
(\mathrm{km} / \mathrm{s})\end{array}$ & $\begin{array}{r}V_{1 \mathrm{AU}} \\
(\mathrm{km} / \mathrm{s})\end{array}$ & $\begin{array}{r}V_{S 0} \\
(\mathrm{~km} / \mathrm{s})\end{array}$ & $\alpha$ & Location & $\begin{array}{l}\xi \\
\left({ }^{\circ}\right)\end{array}$ & $\begin{array}{c}\mathrm{R} \\
(\mathrm{AU})\end{array}$ & $\begin{array}{c}D \\
(\mathrm{AU})\end{array}$ & $\begin{array}{l}\theta_{0} \\
\left(^{\circ}\right)\end{array}$ & $A R$ & $\begin{array}{c}\beta \\
\left(^{\circ}\right)\end{array}$ & $C_{1}$ & $\rho$ & \\
\hline 29 April 1998 & S18E20 & 1374 & 779 & 843 & 0 & N40E21 & 58 & 0.69 & 0.06 & 46 & $\sim 1$ & & 6.3 & 0.92 & \\
\hline 24 Aug 1998 & N35E09 & (N/A) & 1273 & 1064 & 1.2 & N09E59 & 52 & 0.75 & 0.02 & 160 & 0.1 & -4 & 9.6 & 0.78 & [1] \\
\hline 13 April 1999 & (N/A) & 291 & 521 & 506 & 0.9 & S07E16 & & 0.58 & 0.17 & 54 & 0.32 & 48 & 2.4 & 0.57 & \\
\hline 19 July 1999 & N18E59 & 719 & (N/A) & 851 & 5.5 & N28E22 & 35 & 1.37 & 0.35 & 31 & $\sim 1$ & & 9.1 & 0.93 & \\
\hline 17 Aug 1999 & N26E35 & 776 & 740 & 710 & 0.7 & N23E08 & 25 & 0.67 & 0.09 & 67 & 0.31 & -54 & 6.7 & 0.51 & \\
\hline 20 Sep 1999 & (N/A) & 604 & 762 & 824 & 2.8 & S35E04 & & 0.90 & 0.06 & 56 & 0.75 & -25 & 12 & 0.85 & [5] \\
\hline 2 June 2000 & N16E60 & 731 & 958 & 762 & 0 & S15E35 & 40 & 0.57 & 0.12 & 36 & $\sim 1$ & & 4.7 & 0.87 & \\
\hline 6 June 2000 & N20E18 & 1119 & 995 & 838 & 1 & N05W21 & 41 & 0.70 & 0.03 & 69 & 0.29 & 9 & 9.2 & 0.53 & \\
\hline 10 July 2000 & N18E49 & 1352 & 693 & 874 & 2.9 & N13E21 & 27 & 0.63 & 0.07 & 61 & $\sim 1$ & & 3.8 & 0.79 & [3] \\
\hline 11 July 2000 & N18E27 & 1078 & (N/A) & 806 & 0 & S19E37 & 38 & 0.74 & 0.08 & 18 & $\sim 1$ & & 6.9 & 0.84 & [3] \\
\hline 12 July 2000 & N17E27 & 1124 & 785 & 1108 & 5.5 & N02E31 & 16 & 1.08 & 0.21 & 224 & 0.03 & -1 & 5.2 & 0.81 & [3] \\
\hline 14 July 2000 & N22W07 & 1674 & 1473 & 1540 & 3.2 & N11W17 & 15 & 0.64 & 0.13 & 130 & 0.15 & 27 & 6.3 & 0.76 & [2] \\
\hline 10 April 2001 & S23W09 & 2411 & 1287 & 1553 & 2.8 & N08W30 & 37 & 0.81 & 0.09 & 72 & 0.04 & 10 & 9.7 & 0.63 & \\
\hline 25 Aug 2001 & S17E34 & 1433 & 813 & 836 & 0 & N01W11 & 48 & 0.69 & 0.05 & 68 & 0.3 & -8 & 5 & 0.77 & \\
\hline 4 Nov 2001 & N06W18 & 1810 & 1240 & 1295 & 3.6 & S16E09 & 35 & 1.04 & 0.01 & 123 & $\sim 1$ & & 7.9 & 0.71 & \\
\hline 28 Oct 2003 & S16E08 & 2459 & 2185 & 1116 & 3.0 & N15E15 & 32 & 0.42 & 0.14 & 111 & 0.28 & -33 & 4.7 & 0.62 & [4] \\
\hline
\end{tabular}

to be formed provided that the slow wind is distributed in a zonal region with a small latitude width. The formation of an arch-like density structure under a condition of the bimodal solar wind was demonstrated by a 3-dimensional magnetohydrodynamic (MHD) model calculation of CME propagation (Odstrcil et al., 2005). From examination of the solar wind speed data obtained from our IPS observations, we found that the direction of maximum angular extent of the loop-like structure was not inconsistent with the region of slow solar wind (e.g. Tokumaru et al., 2003a).

Nevertheless, it should be mentioned that clear evidence suggesting another origin for $\Delta N_{e}$ enhancements has been found from our IPS observations for the particular CME event of 28 October 2003 (Tokumaru et al., 2005a, b). For this case, a loop-like high- $\Delta N_{e}$ structure propagating at a speed significantly slower than the IP shock speed was identified from the model fitting analysis of our g-value data (see Fig. 4d and Table 1; Tokumaru et al., 2005b), and it was found to be in good agreement with simultaneous observations made with the Solar Mass Ejection Imager (Jackson et al., 2006). We consider that the loop structure identified for this event is likely to represent dense material ejected from the corona. Such events are quite rare, and little is known about their physical properties. Therefore, we need to explore the coronal ejecta origin event further.

The radial evolution of CME propagation speeds is another interesting feature revealed from our IPS study. The dynamics of CMEs in the solar wind is thought to be dominated by an interaction with the ambient solar wind plasma (e.g. Vršnak, 2001), and a pronounced deceleration of fast CMEs is expected in the slow wind region. From a recent study using our IPS observations, the CME speed evolution was found to correlate with the ambient solar wind condition (Yamashita et al., $2006^{1}$ ). In this study, the shell-shaped model was fit to the g-value data to determine CME speeds in the solar wind, and the radial variation of propagation speeds between the corona and the Earth orbit was derived for 9 halo-CME events by combining IPS data with LASCO and in situ observations. The results indicated that the deceleration rate of fast CMEs inversely correlated with the initial speed difference between the CME and the ambient solar wind. We need to confirm this conclusion from a more extensive survey including the elongated CME events in the future. Recently, another important aspect of the CME speed evolution was revealed from an extensive survey using Ooty IPS observations (Manoharan, 2006). It was found from this study that the radial dependence of the CME speed was composed of two power-laws: one that was flat at distances up to $\sim 80 R_{S}$ and one with a steeper slope beyond this distance. The index of the flat portion appeared to be independent of the initial speed of the CME, while that of the steeper portion showed

\footnotetext{
${ }^{1}$ Yamashita, M., Tokumaru, M., Kojima, M., and Fujiki, K.: Radial dependence of CME propagation speed in interplanetary space, J. Geophys. Res., submitted, 2006.
} 
a systematic change with the CME speed. This speed evolution was ascribed to a transition of the propagation dynamics from the one dominated by the CME's initial energy to that dominated by the drag force. This two-step deceleration model may account for some CMEs which showed insignificant deceleration. In addition, the CME speed may be depend on the global CME structure. The relation between the CME structure and the speed evolution will be carefully investigated in a forthcoming study.

Acknowledgements. This work is supported by the Japan Society for the Promotion of Science (grant 16340147). The IPS observations were carried out under the solar wind program of the Solar-Terrestrial Environment Laboratory (STEL) of Nagoya University. SOHO is a project involving international cooperation between ESA and NASA. The SOHO/LASCO CME catalog is generated and maintained by the Center for Solar Physics and Space Weather, Catholic University of America, in cooperation with the Naval Research Laboratory and NASA. We thank the National Geophysical data Center (the Space Physics Interactive Data Resource, SPIDR) and the ACE Science Center for providing GOES and ACE data, respectively, via the web sites. We also would like to thank M. Bird for his helpful comments.

Edited by: A. C. L. Chian

Reviewed by: M. Bird and J. Padmanabhan

\section{References}

Armstrong, J. W. and Coles, W. A.: Interplanetary scintillation of PSR0531+21 at $74 \mathrm{MHz}$, Astrophys. J., 220, 346-352, 1978.

Asai, K., Ishida, Y., Kojima, M., Maruyama, K., Misawa, H., and Yoshimi, N.: Multi-station system for solar wind observations using the interplanetary scintillation method, J. Geomag. Geoelectr., 47, 1107-1112, 1995.

Borrini, G., Gosling, G. T., Bame, S. J., and Feldman, W. C.: An analysis of shock wave disturbances observed at $1 \mathrm{AU}$ from 1971 through 1978, J. Geophys. Res., 87, 4365-4373, 1982.

Brueckner, G. E., Howard, R. A., Koomen, M. J., et al.: The large angle spectroscopic coronagraph (LASCO), Sol. Phys., 162, 357-402, 1995.

Dennison, P. A. and Hewish, A.: The solar wind outside of the plane of the ecliptic, Nature, 213, 343-346, 1967.

Gapper, G. R., Hewish, A., Purvis, A., and Duffet-Smith, P. J.: Observing interplanetary disturbances from the ground, Nature, 296, 633-636, 1982.

Gosling, J. T.: The solar flare myth, J. Geophys. Res., 98, $18937-$ 18 949, 1993.

Hewish, A.: A user's guide to scintillation, J. Atmos. Terr. Phys., 51, 743-750,1989.

Hewish, A., Scott, P. F., and Willis, D.: Interplanetary scintillation of small-diameter radio sources, Nature, 203, 1214-1217, 1964.

Hewish, A., and Symonds, M. D.: Radio investigation of the solar plasma, Planet. Space Sci., 17, 313-320, 1969.

Jackson, B. V., Buffington, A., Hick, P. P., Wang, X., and Webb, D.: Preliminary 3D analysis of the heliospheric response to the 28 October 2003 CME using SMEI white-light observations, J. Geophys. Res., in press, 2006.
Kojima, M. and Kakinuma, T.: Solar cycle dependence of global distribution of solar wind speed, Space Sci. Rev., 53, 173-222, 1990.

Lindsay, G. M., Russell, C. T., and Luhmann, J. G.: Coronal mass ejection and stream interaction region characteristics and their potential geomagnetic effectiveness, J. Geophys. Res., 100, 16999-17 013, 1995.

Luhmann, J. G.: CMEs and Space weather, in Coronal mass ejections, Geophys. Monogr. Ser., Vol. 99, edited by: Crooker, N., Joselyn, J. A., and Feynman, J., AGU, Washington, D.C., 291299, 1997.

Manoharan, P. K., Tokumaru, M., Pic, M., Subramanian, S., Ipavich, F. M., Schenk, K., Kaiser, M. L., Lepping, R. P., and Vourlidas, A.: Coronal mass ejection of 14 July 2000 flare event: Imaging from near-Sun to Earth environment, Astrophys. J., 559, 1180-1189, 2001.

Manoharan, P. K.: Evolution of coronal mass ejections in the inner heliosphere: A study using white-light and scitillation images, Sol. Phys., in press, 2006.

Marubashi, K.: The space weather forecast program, Space Sci. Rev., 51, 197-214, 1989.

McComas, D. J., Bame, S. J., Barker, P. L., Feldman, W. C., Phillips, J. L., Riley, P., and Griffee, J. W.: Solar wind electron proton alpha monitor (SWEPAM) for the Adanced Composition Explorer, Space Sci. Rev., 86, 563-612, 1998.

Moore, V. and Harrison, R. A.: A characterization of discrete solar wind events detected by interplanetary scintillation mapping, J. Geophys. Res., 99, 27-33, 1994.

Odstrcil, D., Pizzo, V. J., and Arge, C. N.: Propagation of the 12 May 1997 interplanetary coronal mass ejection in evolving solar wind structures, J. Geophys. Res., 110, A02106, doi:10.1029/2004JA010745, 2005.

Readhead, A. C. S., Kemp, M. C., and Hewish, A.: The spectrum of small-scale density fluctuations in the solar wind, Mon. Not. R. Astro. Soc., 185, 207-225, 1978.

Reames, D. V.: Particle acceleration at the Sun and in the heliosphere, Space Sci. Rev., 90, 413-491, 1999.

Smart, D. F. and Shea, M. A.: A simplified model for timing the arrival of solar flare-initiated shocks, J. Geophys. Res., 90, 183190, 1985.

St. Cyr, O. C., Howard, R. A., Sheeley Jr., N. R., Plunkett, S. P., Michels, D. J., Paswaters, S. E., Koomen, M. J., Simnett, G. M., Thompson, B. J., Gurman, J. B., Schwenn, R., Webb, D. F., Hildner, E., and Lamy, P. L.: Properties of coronal mass ejections: SOHO LASCO observations from January 1996 to June 1998, J. Geophys. Res., 105, 18 169-18 185, 2000.

Tappin, S. J.: Numerical modeling of scintillation variations from interplanetary disturbances, Planet. Space Sci., 35, 271-283, 1987.

Tokumaru, M., Kojima, M., Fujiki, K., and Yokobe, A.: Threedimensional propagation of interplanetary disturbances detected with radio scintillation measurements at $327 \mathrm{MHz}$, J. Geophys. Res., 105, 10 435-10 453, 2000.

Tokumaru, M., Kojima, M., Fujiki, K., Yamashita, M., and Yokobe, A.: Toroidal-shaped interplanetary disturbance associated with the halo coronal mass ejection event on 14 July 2000, J. Geophys. Res., 108, 1220, doi:10.1029/2002JA009574, 2003a.

Tokumaru, M., Kojima, M., Fujiki, K., and Yamashita, M.: Global structure of interplanetary coronal mass ejections retrieved from 
the model fitting analysis of radio scintillation observations, Proceedings of 10th international conference on solar wind, AIP Conference Proceedings Vol. 679, 729-732, 2003 b.

Tokumaru, M., Kojima, M., Fujiki, K., Yamashita, M., and Baba, D.: Interplanetary consequences caused by the extremely intense solar activity during October-November 2003, J. Geophys. Res., 110, A01109, doi:10.1029/2004JA010656, 2005a.

Tokumaru, M., Kojima, M., Fujiki, K., Yamashita, M., and Jackson, B. V.: Interplanetary scintillation measurements of a transient solar wind stream associated with the 2003 October 28 fullhalo coronal mass ejection, Proceedings of URSI GA 2005, J05P2(0193), 2005b.

Tokumaru, M., Yamashita, M., Kojima, M., Fujiki, K., and Nakagawa, T.: Reconstructed global feature of an interplanetary disturbance for the full-halo coronal mass ejection event on 20 September 1999, Adv. Space Res., in press, 2005c.
Vlasov, V. I.: Radio imagery of the turbulent interplanetary plasma, Sov. Astron., 23, 55-59, 1979.

Vrs̆nak, B.: Deceleration of coronal mass ejections, Sol. Phys., 202, 173-189, 2001.

Watanabe, T. and Schwenn, R.: Large-scale propagation properties of interplanetary disturbances revealed from IPS and spacecraft observations, Space Sci. Rev., 51, 147-173, 1989.

Woo, R. and Armstrong, J. W.: Spacecraft radio scattering observations of the power spectrum of electron density fluctuations in the solar wind, J. Geophys. Res., 84, 7288-7296, 1979.

Yashiro, S., Gopalswamy, N., Michalek, G., St. Cyr, O. C., Plunkett, S. P., Rich, N. B., and Howard, R. A.: A catalog of white light coronal mass ejections observed by the SOHO spacecraft, J. Geophys. Res., 109, A07105, doi:10.1029/2003JA010282, 2004.

Young, A. T.: Interpretation of interplanetary scintillation, Astrophys. J., 168, 543-562, 1971. 